

University of Kentucky

UKnowledge

Sanders-Brown Center on Aging Faculty

Publications

Aging

$11-2017$

\title{
Cerebral Amyloid Angiopathy in Down Syndrome and Sporadic and Autosomal-Dominant Alzheimer's Disease
}

\author{
María Carmona-Iragui \\ Universitat Autònoma de Barcelona, Spain \\ Mircea Balasa \\ Trinity College Dublin, Ireland \\ Bessy Benejam \\ Barcelona Down Medical Center, Spain \\ Daniel Alcolea \\ Universitat Autònoma de Barcelona, Spain

\section{Susana Fernández} \\ Barcelona Down Medical Center, Spain \\ Follow this and additional works at: https://uknowledge.uky.edu/sbcoa_facpub \\ Part of the Diseases Commons, Genetics and Genomics Commons, and the Neurosciences Commons \\ See next page for additional authors \\ Right click to open a feedback form in a new tab to let us know how this document benefits you.
}

\section{Repository Citation}

Carmona-Iragui, María; Balasa, Mircea; Benejam, Bessy; Alcolea, Daniel; Fernández, Susana; Videla, Laura; Sala, Isabel; Sánchez-Saudinós, María Belén; Morenas-Rodriguez, Estrella; Ribosa-Nogué, Roser; Illán-Gala, Ignacio; Gonzalez-Ortiz, Sofía; Clarimón, Jordi; Schmitt, Frederick A.; Powell, David K.; Bosch, Beatriz; Lladó, Albert; Rafii, Michael S.; Head, Elizabeth; Molinuevo, José Luis; Blesa, Rafael; Videla, Sebastián; Lleó, Alberto; Sánchez-Valle, Raquel; and Fortea, Juan, "Cerebral Amyloid Angiopathy in Down Syndrome and Sporadic and Autosomal-Dominant Alzheimer's Disease" (2017). Sanders-Brown Center on Aging Faculty Publications. 117.

https://uknowledge.uky.edu/sbcoa_facpub/117

This Article is brought to you for free and open access by the Aging at UKnowledge. It has been accepted for inclusion in Sanders-Brown Center on Aging Faculty Publications by an authorized administrator of UKnowledge. For more information, please contact UKnowledge@lsv.uky.edu. 


\section{Cerebral Amyloid Angiopathy in Down Syndrome and Sporadic and Autosomal- Dominant Alzheimer's Disease}

Digital Object Identifier (DOI)

https://doi.org/10.1016/j.jalz.2017.03.007

\section{Notes/Citation Information}

Published in Alzheimer's \& Dementia: Journal of the Alzheimer's Association, v. 13, issue 11, p. 1251-1260.

(c) 2017 Published by Elsevier Inc. on behalf of the Alzheimer's Association.

This manuscript version is made available under the CC-BY-NC-ND 4.0 license http://creativecommons.org/licenses/by-nc-nd/4.0/.

The document available for download is the author's post-peer-review final draft of the article.

\section{Authors}

María Carmona-Iragui, Mircea Balasa, Bessy Benejam, Daniel Alcolea, Susana Fernández, Laura Videla, Isabel Sala, María Belén Sánchez-Saudinós, Estrella Morenas-Rodriguez, Roser Ribosa-Nogué, Ignacio Illán-Gala, Sofía Gonzalez-Ortiz, Jordi Clarimón, Frederick A. Schmitt, David K. Powell, Beatriz Bosch, Albert Lladó, Michael S. Rafii, Elizabeth Head, José Luis Molinuevo, Rafael Blesa, Sebastián Videla, Alberto Lleó, Raquel Sánchez-Valle, and Juan Fortea 


\title{
Cerebral amyloid angiopathy in Down syndrome and sporadic and autosomal dominant Alzheimer's disease
}

\author{
María Carmona-Iragui, MD ${ }^{a, b, c, d, 1}$, Mircea Balasa, PhD ${ }^{\mathrm{d}, \mathrm{e}, 1}$, Bessy Benejam, MSc ${ }^{\mathrm{b}}$, Daniel \\ Alcolea, MD ${ }^{a, c}$, Susana Fernández, MD $^{b}$, Laura Videla, MSc $^{b}$, Isabel Sala, PhD $^{a, c}$, María \\ Belén Sánchez-Saudinos, MSc ${ }^{a, c}$, Estrella Morenas-Rodriguez, MD ${ }^{a, c}$, Roser Ribosa- \\ Nogué, MD ${ }^{a, c}$, Ignacio Illán-Gala, MD ${ }^{a, c}$, Sofía Gonzalez-Ortiz, MD ${ }^{f}$, Jordi Clarimón, PhD $^{a, c}$, \\ Frederick Schmitt, PhD ${ }^{g}$, David K Powell, PhDg, Beatriz Bosch, PhD ${ }^{d}$, Albert Lladó, PhD ${ }^{d}$, \\ Michael Rafii, MD ${ }^{h}$, Elizabeth Head, PhDg, José Luis Molinuevo, PhD ${ }^{d, i}$, Rafael Blesa, MD ${ }^{a, c}$, \\ Sebastián Videla, MD ${ }^{\mathrm{b}, \mathrm{j}}$, Alberto Lleó, MD ${ }^{\mathrm{a}, \mathrm{c}, 2}$, Raquel Sánchez-Valle, PhD $^{\mathrm{d}, 2}$, and Juan \\ Fortea, MDa,b,c,2
}

aMemory Unit, Department of Neurology, Hospital de la Santa Creu i Sant Pau- Biomedical Research Institute Sant Pau-Universitat Autònoma de Barcelona. Sant Antoni Mํㅡ Claret 167, 08025 Barcelona, Spain barcelona Down Medical Center. Fundació Catalana de Síndrome de Down. Compte Borrell 201-203, 08029, Barcelona, Spain 'Centro de Investigación Biomédica en Red de Enfermedades Neurodegenerativas. CIBERNED. Valderrebollo 5, 28031, Madrid, Spain ${ }^{d}$ Global Brain Health Institute, Trinity College Dublin. College Green, Dublin 2, Ireland eAlzheimer's Disease and other cognitive disorders unit, Neurology Department, Hospital Clínic, Institut d'Investigació Biomèdica August Pi i Sunyer (IDIBAPS). Villarroel 170, 08036, Barcelona, Spain fDepartment of Radiology, Hospital del Mar- Universitat Autònoma de Barcelona. Paseo Marítimo 25-29, 08003, Barcelona, Spain 9Sanders-Brown Center on Aging, University of Kentucky, Lexington. KY 40536, USA hAdult Down Syndrome Clinic, Department of Neuroscience, University of California, 4510 Executive Drive, San Diego CA 92121 and Alzheimer's Therapeutic Research Institute University of Southern California, 9860 Mesa Rim Rd, San Diego CA 92121, USA 'BarcelonaBeta Brain research Center, Fundació Pasqual Maragall, Universitat Pompeu Fabra. Wellington 30, 08005, Barcelona, Spain iDepartment of Experimental and Health Sciences. Faculty of Health and Life Sciences. Universitat Pompeu Fabra. Doctor Aiguader 88, 08003, Barcelona, Spain

\section{Abstract}

Introduction-We aimed to investigate if cerebral amyloid angiopathy (CAA) is more frequent in genetically determined than in sporadic early onset forms of Alzheimer disease (EOAD).

Methods-Neuroimaging features of CAA, $A P O E$, and cerebrospinal fluid-A $\beta 40$ levels were studied in subjects with Down syndrome (DS, $n=117$ ), autosomal dominant AD (ADAD, n=29), sporadic EOAD $(n=42)$, and healthy controls $(n=68)$.

"Corresponding authors: Juan Fortea Ormaechea, Memory Unit, Department of Neurology, Hospital de la Santa Creu y Sant Pau. Sant

Antoni María Claret, 167. 08025. Barcelona. Spain. Phone number: (34) 935565986. Fax: (34) 935565602. jfortea@ santpau.cat.

1 These authors contributed equally to the manuscript.

2 These authors share senior authorship. 
Results-CAA was present in $31 \%, 38 \%$ and $12 \%$ of cognitively impaired DS, symptomatic ADAD, and sporadic EOAD subjects, and in $13 \%$ and $4 \%$ of cognitively unimpaired DS individuals and healthy controls, respectively. $A P O E-\varepsilon 4$ genotype was borderline significantly associated with CAA in sporadic EOAD ( $p=0.06)$, but not with DS or ADAD. There were no differences in A $\beta 40$ levels between groups or between subjects with and without CAA.

Discussion-CAA is more frequently found in genetically determined AD than in sporadic EOAD. Cerebrospinal fluid-A $\beta 40$ levels are not a useful biomarker for CAA in AD.

\section{Keywords}

Cerebral amyloid angiopathy; sporadic early onset Alzheimer disease; autosomal dominant Alzheimer disease; Down syndrome; neuroimaging; cerebrospinal fluid biomarkers

\section{Introduction}

Most cases of Alzheimer's disease are sporadic, and caused by complex interactions between genetic and environmental factors. In approximately $5 \%$ of cases, Alzheimer's disease can present clinically before the age of 65 (early-onset Alzheimer's disease-EOAD) [1]. These patients frequently present with non-amnestic phenotypes and faster clinical decline than older sporadic Alzheimer's disease cases [1]. In 0.1-0.5\% of cases, Alzheimer's disease is transmitted with an autosomal dominant pattern of inheritance (ADAD) due to the presence of mutations in presenilin 1 (PSEN1), presenilin 2 (PSEN2), or amyloid precursor protein $(A P P)$ genes [2]. Down syndrome (DS) is also recognized as a form of genetically determined AD, mainly caused by the $A P P$ gene triplication [2]. Despite the different genetic background, the Alzheimer's disease neuropathological findings in sporadic EOAD, ADAD and DS are very similar [3,4].

Cerebral Amyloid Angiopathy (CAA) is a major cause of lobar intracerebral hemorrhage (ICH) in the elderly and is present in up to $90 \%$ of Alzheimer's disease brains at autopsy [3]. Previous neuropathological studies have suggested a more severe CAA in ADAD than in sporadic Alzheimer's disease [4]. CAA in some APP mutations or duplication carriers drives the clinical presentation [4], and is also consistently observed in subjects with DS [5]. The modified Boston criteria for CAA-related hemorrhage (mBCAA) have been validated to attribute in vivo an ICH to CAA based on several neuroimaging features, and are frequently used in clinical practice [6]. There are no previous studies systematically assessing the CAA neuroimaging features in DS and ADAD.

$A \beta 40$ is the major form of $A \beta$ deposited in the vessel walls in individuals with CAA. Low levels of $A \beta 40$ and $A \beta 42$ have been found in the CSF of subjects with sporadic CAA [7]. However, scarce and contradictory data are available about the CAA CSF biomarker profile in sporadic Alzheimer's disease patients [8-10], and no previous studies have assessed this profile in DS or ADAD. Moreover, the APOE- 44 genotype is a risk factor for both sporadic Alzheimer's disease and sporadic CAA [11], as it increases A $\beta$ deposition in both the parenchyma and blood vessels [12]. However, the effect of the APOE genotype in Alzheimer's disease dementia within DS and ADAD is controversial, and there are no studies assessing the influence of the $A P O E$ genotype on CAA in ADAD or DS [13]. 
The differences in the CAA neuroimaging features and CSF biomarkers profile in the different forms of Alzheimer's disease are thus, not established. Our primary objective was to determine the CAA presence assessing the fulfillment of the mBCAA and the CSF A $\beta 40$ levels in three different Alzheimer's disease populations: DS, ADAD and EOAD. We hypothesized that patients with genetically determined Alzheimer's disease would have more CAA neuroimaging and biochemical features than EOAD.

\section{Materials and methods}

\section{Study design and participants}

A total of 256 subjects were recruited from 5 centers: Hospital of Sant Pau, Hospital Clínic de Barcelona, and Barcelona Down Medical Center in Barcelona, Spain; and the SandersBrown Center on Aging in Kentucky and the Down Syndrome Biomarker Initiative (DSBI) project in San Diego, United States of America. Four study groups were evaluated: EOAD, ADAD, DS, and healthy controls (HC).

EOAD (N=42)-Patients were recruited at the Memory Unit of Hospital de Sant Pau from the Sant Pau Initiative on Neurodegeneration (Barcelona SPIN cohort) [14]. We used the International Working Group-2 diagnostic criteria for $\mathrm{AD}$ with in vivo evidence of $\mathrm{AD}$ based on CSF biomarkers [2]. This group included 19 individuals with prodromal AD (p-EOAD) and 23 subjects with probable AD dementia (d-EOAD).

ADAD (N=29)-Participants were recruited from the Genetic counseling program for familial dementias (PICOGEN) at the Hospital Clínic de Barcelona [15]. Fifteen symptomatic carriers (CDR 20.5) carrying 9 different PSEN1 mutations (M139T, S169P, L173F, G209E, L235R, K239N, L282R, L286P, I439S) and one symptomatic carrier of the $A P P$ I716T mutation were included. The symptomatic carriers were further classified as prodromal ADAD (pAD-ADAD, $n=5$ ) and ADAD dementia (dADAD, $n=11$ ). Twelve presymptomatic mutation carriers (CDR=0) carrying 7 different PSEN1 mutations (M139T, S169P, L173F, R220G, K239N, L282R, I439S) and one pre-symptomatic carrier of $A P P$ I716T were labeled as asymptomatic ADAD. We used the IWG2 diagnostic criteria for AD [2].

DS (N=117)-Adults with DS were recruited from three centers, the Down Alzheimer Barcelona Neuroimaging Initiative (DABNI) in the Barcelona Down Medical Center [16]; the Sanders-Brown Center on Aging; and the DSBI pilot project [17]. Adapted neuropsychological batteries (detailed in the Appendix section), covering all cognitive domains classified DS subjects into "without cognitive decline" (asymptomatic DS, N=91), prodromal AD (pAD-DS, N=13), and AD dementia (dAD-DS, N=13). pAD-DS and dADDS were also labeled as symptomatic DS.

Healthy controls ( $\mathbf{N}=68)$-Participants were recruited at Hospital de Sant Pau ( $\mathrm{n}=60)$ and Hospital Clínic de Barcelona ( $\mathrm{n}=8$ ) enrolled among patients' caregivers. They did not have cognitive complaints, scored 0 on CDR, had normal neuropsychological evaluation, and normal core Alzheimer's disease CSF biomarkers [18,19]. 
Procedures

Medical records were reviewed for potential confounders and effect modifiers: age, sex, and presence of arterial hypertension, neuropsychological information on disease severity (MMSE for EOAD and ADAD and the Cambridge Examination for mental Disorders of Older People with DS and Others with Intellectual Disabilities-Cognitive Scale CAMCOG-DS scale for DS) were recorded and sent to the coordinating center (Hospital de Sant Pau) with the CSF biomarkers (A $\beta 42, A \beta 40)$ and neuroimaging data.

The study was approved by the local Ethics Committees following the ethical standards recommended by the Helsinki Declaration. All participants and/or their caregivers gave their written informed consent.

\section{Neuroimaging assessments}

The inclusion criteria for all participants included a 1.5 or 3T MRI scan including T2*-GRE or SWI, axial fluid attenuated inversion recovery (FLAIR) and coronal T1-weighted sequences in the five centers involved GRE or SWI sequences were assessed for the presence of the main CAA neuroimaging features: localization and number of lobar microbleeds, presence of cortical superficial siderosis (cSS), and lobar ICH. We evaluated the fulfillment of mBCAA in all participants regardless of the age criterion included in the criteria set ( $>55$ years) [6]. White matter hyperintensites $(\mathrm{WMH})$ were semi-quantitatively assessed in FLAIR sequences according to the Fazekas score [20]. MTA was evaluated in coronal T1-weighted images trough the Scheltens scale [21]. MTA was scored bilaterally and the highest score was considered for the analyses.

The radiological evaluation was performed by two raters (either MCI or MB; neurologists with expertise in cognitive disorders and SG; neuroradiologist) blinded to the clinical data. Inter-rater reliability was above $90 \%$ and discrepancies within ratings were solved by consensus.

\section{CSF biomarkers and APOE genotype}

The inclusion criteria for EOAD and healthy controls included CSF data, but not for ADAD and DS. Details of analysis are described elsewhere $[14,18]$. In short, commercially available ELISA kits were used to determine CSF-A 440 and CSF-A $\beta 42$ levels (Millipore and Fujirebio-Europe, respectively), following the manufacturers' recommendations.

APOE genotyping was performed by PCR amplification of the exon four fragment containing the two polymorphisms (rs429358 and rs7412) that encode the three common $A P O E$ isoforms. The following oligonucleotides: APOE-F: $5^{\prime}-$ ACTGGAGGAACAACTGACCC-3' and APOE-R: 5' $^{\prime}$ CTGCCCATCTCCTCCATC-3', were used and final PCR products were purified and Sanger sequenced using BigDye terminator chemistry (Applied Biosystems). Sequences were run on an Applied Biosystems ${ }^{\circledR} 3130$ Genetic Analyzer and resulting elecropherograms were visually inspected using Sequencher (version 4.1, Gene Codes Corp.). 


\section{Statistical analysis}

Statistical analyses were performed with the Statistical Package for the Social Sciences v19 software (IBM corp. http://www-01.ibm.com/software/es/analytics/spss/). The primary objectives of this study were to compare across groups the frequency of the mBCAA and the CSF-A $\beta 40$ levels and were analyzed with the exact Fisher and Mann-Whitney test respectively.

The secondary objectives were to assess the white matter lesions measured by the Fazekas scale and the hippocampal atrophy measured by the Scheltens scale and were analyzed with the exact Fisher test. Spearman correlation coefficients were calculated between age, clinical stage, hippocampal atrophy and white matter lesions and the different study groups. With the purpose of improving the statistical power, prodromal and demented groups in EOAD, ADAD and DS were merged when analyzed. All significance tests were two-sided with the statistical significance set at $5 \%$.

\section{Results}

Table 1 displays the demographics, clinical features, CSF data, and $A P O E$ genotype of the participants. CSF data were available in $71 \%(\mathrm{~N}=182)$ of the subjects (including all healthy controls and patients with EOAD). Symptomatic ADAD and symptomatic DS subjects were younger than patients with $\operatorname{EOAD}(48.4,54.4,61.1$ years of age respectively; $p<0.001)$.

The $A P O E-\varepsilon 4$ allele frequency was higher in EOAD than in any other group. However, no differences were observed between symptomatic or asymptomatic subjects within the ADAD or DS groups.

Table 2 shows the neuroimaging results across the different groups. The fulfillment of the mBCAA criteria was more frequent in symptomatic $\mathrm{DS}, 31 \%(\mathrm{~N}=8)$, and in symptomatic ADAD, 38\% ( $\mathrm{N}=6)$, than in EOAD 24\% ( $\mathrm{N}=19)(p=0.055$ and 0.026 respectively). When present, the most frequent CAA neuroimaging features were lobar microbleeds in $91.2 \%$ $(\mathrm{N}=31)$, followed by cSS in $29.4 \%(\mathrm{~N}=10)$ and ICH in $8.8 \%(\mathrm{~N}=3)$. All three features were more frequent in the symptomatic than in asymptomatic subjects within all groups (Table 2).

The symptomatic ADAD and DS groups had a higher proportion of lobar microbleeds than the EOAD group ( $p=0.02, p=0.046$ respectively). $\mathrm{cSS}$ and ICH were statistically associated $(p=0.004)$. In those who had cSS, $20 \%(\mathrm{~N}=2)$ had also an $\mathrm{ICH}$, and cSS was present in $67 \%$ $(\mathrm{N}=2)$ of those with ICH. Symptomatic DS had a higher proportion of subjects with cSS and lobar ICH than the EOAD group, but this difference did not reach statistical significance ( $p=0.056$ ). The position of the mutation (pre or post codon 200) did not significantly impact the presence of lobar microbleeds in PSEN1 carriers (37 vs 25\%, $p=0.4$ ). One of the asymptomatic ADAD subjects included in our study had a massive lobar ICH after recruitment into this study that lead to its dead in a stage of moderately severe dementia.

The mean time lag between MRI and CSF sampling was 5.3 months. Symptomatic participants had lower CSF-A $\beta 42$ levels than asymptomatic subjects within all groups (Figure 1A). No significant differences were detected in CSF-A $\beta 40$ levels between the 
different groups or between symptomatic and asymptomatic subjects within each group (Figure 1B).

All EOAD patients fulfilling the mBCAA were $A P O E-\varepsilon 4$ carriers and the $A P O E$ - $\varepsilon 4$ allele was significantly more frequent in EOAD subjects than in the symptomatic ADAD ( $p=0.015$ ) or symptomatic DS ( $p=0.071)$ subjects fulfilling the mBCAA criteria. In sporadic EOAD, there was a trend for the association between mBCAA fulfillment and APOE-ع4 genotype $(p=0.06)$.

There were no differences in CSF-A $\beta 40$ levels between those subjects who fulfilled the mBCAA criteria and those who did not (or the presence of lobar microbleeds, cSS, or ICH) in any group.

Symptomatic subjects presented higher Fazekas scores than asymptomatic subjects in all groups: EOAD patients had higher Fazekas scores than healthy controls $(p<0.001)$; symptomatic ADAD higher than asymptomatic $\operatorname{ADAD}(p=0.022)$ or $\mathrm{HC}(p=0.05)$; and symptomatic DS higher than asymptomatic DS $(p=0.011)$ and healthy controls $(p<0.001)$ (Figure 2 and table 2).

There was a significant positive correlation between age and Fazekas score in the whole sample ( $\mathrm{r}=0.337, p<0.001)$, in ADAD ( $\mathrm{r}=0.407, p=0.031)$ and in $\mathrm{DS}(\mathrm{r}=0.506, p=0.000)$ groups. This correlation was also found in asymptomatic DS $(\mathrm{r}=0.495, p<0.001)$ and in healthy controls $(\mathrm{r}=0.323, p=0.007)$ (Figure 3).

Age positively correlated with Scheltens scores in the whole sample $(\mathrm{r}=0.229, p<0.001)$, healthy controls ( $\mathrm{r}=0.276, p=0.023)$, ADAD $(\mathrm{r}=0.412, p=0.027)$, asymptomatic $\mathrm{DS}(\mathrm{r}=0.341$, $p=0.001)$, and symptomatic DS ( $\mathrm{r}=0.431, p=0.023)$. The Scheltens scores increased from asymptomatic to symptomatic subjects within each group. Symptomatic DS patients presented higher MTA scores than EOAD and symptomatic ADAD patients $(p<0.001$ in each comparison) and asymptomatic DS subjects higher than $\mathrm{HC}(p<0.001)$. There were no differences in Scheltens scores between EOAD and symptomatic ADAD.

\section{Discussion}

We found that DS and ADAD have a more severe CAA than EOAD as measured by the mBCAA criteria, but CAA did not impact the CSF-A $\beta 40$ levels. The $A P O E-\varepsilon 4$ allele might be associated with CAA in EOAD, but does not seem to have an effect in DS or ADAD.

There are previous studies assessing the prevalence of lobar microbleeds in ADAD and EOAD [22] [23,24], but, to our knowledge, none has specifically assessed and compared the mBCAA between the different AD populations. The mBCAA were more frequent in DS and ADAD, suggesting a more severe CAA, as shown in pathological studies [5,25]. The most frequent CAA neuroimaging feature was the presence of lobar microbleeds, as previously described [26]. The frequency of lobar microbleeds in ADAD (and healthy controls) was in agreement with the literature (ranging from $25 \%$ to $66 \%$ ) [23,24][26], but we found a lower frequency of lobar microbleeds in EOAD (9.5\%) than that reported in late onset AD (20-30\%) [27]. Age might be responsible for this difference. There are no previous studies 
assessing the mBCAA criteria or any of its component neuroimaging features in DS. CAA is also consistently observed in DS pathological studies [5,28], but it had been proposed that other genetic factors in DS might protect these subjects from the ICH [29]. We found 38.5\% of frequency for lobar microbleeds in symptomatic DS and, more importantly, a frequency of $15.4 \%$ for ICH. While this is lower than the reported $30 \%$ prevalence for symptomatic ICH in non-trisomic APP duplication carriers, it is well above the 3\%-3.8\% figure for symptomatic ICH in DS reported in the same study [29]. This discrepancy might be explained because many non-fatal ICH might be unnoticed in DS with AD. Of note, both subjects with DS and ICH on the MRI did not present with ICH-related clinical symptoms.

A significant percentage of symptomatic subjects, nonetheless, did not meet the mBCAA and were free of the CAA associated neuroimaging features. This is in contrast with pathological studies, where CAA is found in up to $90 \%$ of AD brains, suggesting that the available MRI sequences only identify a subset of AD-CAA subjects [23]. The CAA neuroimaging features might thus detect only the most severe cases or, alternatively, they might select different subgroups of patients [23]. In this respect, cSS, although less frequent than lobar microbleeds, was strongly associated with lobar ICH. cSS might be a particular important marker for severe $\mathrm{CAA}$ in $\mathrm{AD}$, as it has been suggested in sporadic CAA [7]. More work in longitudinal studies is needed to confirm the higher risk conferred by cSS for future ICH and cognitive decline.

We also assessed other neuroimaging features associated with CAA, but not included in the mBCAA criteria. Both WMH and MTA are increasingly recognized as core Alzheimer's disease features and as a manifestation of CAA [30]. We found a gradient in WMH extension in all groups [31], but we also found more extended WMH in those subjects fulfilling the mBCAA criteria. WMH also increased with age and in relation with vascular risk factors. We found this correlation also in healthy controls, even though they all had normal core Alzheimer's disease CSF biomarkers and low prevalence of HBP. However, we found a strong correlation between age and WMH in asymptomatic DS despite their younger mean age. This correlation supports the relationship between amyloid deposition and WMH. Not surprisingly, the Scheltens scores increased with symptom severity in all AD populations. Hippocampal atrophy, however, was more severe in DS, even in asymptomatic DS individuals. These results are in agreement with the notion that individuals with DS have smaller hippocampal size from birth, but also show atrophy when Alzheimer's disease develops [17].

Decreased CSF-A $\beta 40$ levels might differentiate sporadic CAA from healthy controls and Alzheimer's disease cases [7]. In our study, nevertheless, CSF-A 440 levels did not discriminate CAA neuroimaging features in any group. This finding could be influenced by the fact that amyloid vascular burden in CAA in ADAD and DS contains not just A $\beta 40$, but also $A \beta 42$. It is difficult to sort out the contribution of vascular $A \beta 42$ deposition from parenchymal plaque deposition except with neuropathological analysis of the post-mortem brain, which was not available in this study $[32,33]$.

To our knowledge, there are only two studies that determine CSF-A 340 in subjects with Alzheimer's disease with and without lobar microbleeds and show conflicting findings 
$[9,10]$. In any case, our results suggest that CSF-A $\beta 40$ levels are not a sensitive biomarker to detect CAA in the context of an Alzheimer's disease process.

The $A P O E-\varepsilon 4$ allele confers a higher risk for CAA in the general population and in Alzheimer's disease [11,12]. We also found a trend for an association between $A P O E-\varepsilon 4$ genotype and CAA in sporadic early onset Alzheimer's disease. Furthermore, all EOAD subjects with CAA neuroimaging features were $A P O E-\varepsilon 4$ carriers. We did not find this relationship in ADAD or DS. The $A P O E-\varepsilon 4$ genotype might be thus associated with CAA in EOAD, but not in DS or ADAD. In ADAD and DS, other genetic factors, such as the type or position of the causing mutation in ADAD, might be more important in predicting CAA [4].

Our findings have potential clinical implications. The mBCAA criteria have not been validated in patients $<55$ years of age. We consider that, at least in ADAD and DS, age should not be an essential requirement for CAA diagnosis. Our results also have substantial implications in Alzheimer's disease clinical trials given the relationship between CAA and amyloid-related neuroimaging abnormalities (ARIA). Vascular amyloid may be a pathophysiological mechanism for ARIA $[34,35]$ and recent studies have shown that after $A \beta$ immunotherapy there is an increase in CAA severity and an increase in lobar microbleeds associated with removal of plaques [36]. Trials targeting A $\beta$ commonly use lobar microbleeds and $A P O E$ genotype to stratify subjects [37]. However, there are no available data on the relationship of CAA neuroimaging abnormalities (and $A P O E$ ) and ARIA in the setting of amyloid-lowering therapy in ADAD and DS. Our data emphasize heterogeneity in prevalence and possibly etiology for CAA, therefore, the recommendations on the exclusions for presence of baseline ARIA-H (microbleeds or hemosiderosis) from sporadic Alzheimer's disease should be taken with caution. On the other hand, the APOE-ع4 genotype is also commonly used to stratify participants given its influence on ARIA [37], however this strategy might not be as important in ADAD or DS. Finally, our data also suggest that the CSF-A $\beta 40$ levels will not be a useful biomarker in these trials.

The higher prevalence of CAA in ADAD and DS might play a role in the conversion to clinical dementia. In sporadic Alzheimer's disease, CAA is an independent contributor to cognitive impairment and can worsen the severity of cognitive dysfunction [38]. Future longitudinal studies are needed to assess the CAA contribution to cognitive impairment in ADAD and DS.

The main strengths of the present study are the relatively large sample size of different rare populations, such as ADAD and DS, as well as the confirmation of the clinical diagnosis with genetics or CSF biomarkers. The study has some limitations. The use of two different imaging techniques is an important limitation when estimating the real prevalence of lobar microbleeds. SWI has a higher sensitivity for hemosiderin, detecting up to 50\% more lobar microbleeds than conventional T2*GRE [39]. However, in our study the ADAD group was exclusively investigated using T2*GRE leading to a possible underestimation of the CAA neuroimaging features in these subjects. Finally, most of the EOAD patients were at a stage of mild dementia and we lack neuropathological data. 
In conclusion, the CAA-associated neuroimaging features are more frequent in adults with DS and in patients with ADAD than in those with EOAD suggesting a more severe CAA pathology. Our study also shows that the CSF-A $\beta 40$ levels are not a reliable biomarker for CAA and that the risk factors for CAA (such as the $A P O E-\varepsilon 4$ genotype) might be different in EOAD and genetically determined Alzheimer's disease. These findings should be taken into account in the design of clinical trials with anti-amyloid therapies in people with ADAD or DS.

\section{Acknowledgments}

We thank the participants and their families for their generosity. We want to acknowledge Laia Muñoz and Raúl Núñez for the laboratory and sample handling.

Funding: This work has been partially supported by research grants from the Carlos III national Institute of Health of Spain (PI13/01532 to RB; PI11/02526 and PI14/01126 to JF; PI08/0036 and PI12/00013 to RSV) jointly funded by Fondo Europeo de Desarrollo Regional (FEDER), Unión Europea, "Una manera de hacer Europa"; Fundació Marató TV3 (project 20141210 to JF); and CIBERNED (Program 1, Alzheimer Disease and other dementias to A.L). This work has also been partially supported by a grant from the Griffols Foundation, the Generalitat de Catalunya (2014SGR-0235), and by the Fundació Catalana de Síndrome de Down. Funding to support imaging studies in DS studies from UKY to EH/FAS/DP is from NIH NICHD R01HD064993. MCI is supported by Contrato de formación en Investigación post Formación Sanitaria Especializada Río Hortega (CM14/00029) from the Carlos III national Institute of Health of Spain. IIG is supported by i-PFIS grant (IF15/00060) from the Fondo de Investigaciones Sanitarias, Carlos III national Institute of Health of Spain.

\section{Appendix}

Neuropsychological batteries assessed in the Down syndrome group.

Each participant received an annual physical and neurological examination. Diagnostic was established after a clinical consensus performed by the clinical neurologist and the neuropsychologist.

- $\quad$ Neuropsychological battery from the Down Alzheimer Barcelona Neuroimaging Initiative (DABNI), Spain.

- $\quad$ Kauffman Brief Intelligence Test

- $\quad$ CAMDEX-DS (informant interview) and CAMCOG-DS (subject cognitive assessment)

- $\quad$ Clued recall test (Free and cued immediate and delayed recall)

- $\quad$ Digit span (forward/ backward)

- $\quad$ Picture cancellation task (Devenny)

- Barcelona Test (assessment of limb apràxia-pantomime of intransitive gestures)

- $\quad$ Cats and dogs tests

- $\quad$ Verbal fluency (animals)

- $\quad$ Abstract thinking (Barcelona test) 
- $\quad$ Mental State examination- DS (MEC-DS), screening instrument, not validated

- $\quad$ Neuropsychiatric inventory (12 item NPI)

- $\quad$ Fundació Catalana Sindrome de Down Functional Scale, not validated

- $\quad$ Dementia questionnaire for people with intellectual disabilities (DMR)

- $\quad$ Neuropsychological battery from the Sanders-Brown Center on Aging in Kentucky, USA.

- $\quad$ Kauffman Brief Intelligence Test

- $\quad$ Severe Impairment Battery

- $\quad$ Brief Praxis Test

- $\quad$ Fuld Object Memory Evaluation

- $\quad$ Peabody Picture Vocabulary Test (4th ed.)

- $\quad$ Children's Memory Scale: Dot Locations

- $\quad$ Category verbal fluency

- $\quad$ Beery Visual Motor Integration

- $\quad$ Neuropsychiatric inventory (NPI)

- $\quad$ WISC-R Block Design

- Vineland Adaptive Behavior Scale

- $\quad$ Behavioral Rating of Executive Functions (BRIEF)

- $\quad$ Adaptive Behavior Assessment System (ABAS)

- $\quad$ Dementia Questionnaire for people with intellectual disabilities (DMR)

- $\quad$ Neuropsychological battery from the Down Syndrome Biomarker Initiative (DSBI) project in San Diego, USA is detailed in Rafii et al. Front Behav Neurosci. 2015, 14;9:239.

\section{References}

1. Warren JD, Fletcher PD, Golden HL. The paradox of syndromic diversity in Alzheimer disease. Nat Rev Neurol. 2012; 8:451-464. [PubMed: 22801974]

2. Dubois B, Feldman HH, Jacova C, Hampel H, Molinuevo JL, Blennow K, et al. Advancing research diagnostic criteria for Alzheimer's disease: The IWG-2 criteria. Lancet Neurol. 2014; 13:614-629. [PubMed: 24849862]

3. Duyckaerts C, Delatour B, Potier MC. Classification and basic pathology of Alzheimer disease. Acta Neuropathol. 2009; 118:5-36. [PubMed: 19381658]

4. Ringman JM, Monsell S, Ng DW, Zhou Y, Nguyen A, Coppola G, et al. Neuropathology of Autosomal Dominant Alzheimer Disease in the National Alzheimer Coordinating Center Database. J Neuropathol Exp Neurol. 2016; 75:284-290. [PubMed: 26888304] 
5. Wilcock DM, Schmitt FA, Head E. Cerebrovascular contributions to aging and Alzheimer's disease in Down syndrome. Biochim Biophys Acta. 2015; 1862:909-914. [PubMed: 26593849]

6. Linn J, Halpin a, Demaerel P, Ruhland J, Giese aD, Dichgans M, et al. Prevalence of superficial siderosis in patients with cerebral amyloid angiopathy. Neurology. 2010; 74:1346-1350. [PubMed: 20421578]

7. Martínez-Lizana E, Carmona-Iragui M, Alcolea D, Gómez-Choco M, Vilaplana E, SánchezSaudinós MB, et al. Cerebral amyloid angiopathy-related atraumatic convexal subarachnoid hemorrhage: an ARIA before the tsunami. J Cereb Blood Flow Metab. 2015; 35:710-717. [PubMed: 25735919]

8. Shams S, Granberg T, Martola J, Li X, Shams M, Fereshtehnejad SM, et al. Cerebrospinal fluid profiles with increasing number of cerebral microbleeds in a continuum of cognitive impairment. J Cereb Blood Flow Metab. 2016; 36:621-628. [PubMed: 26661151]

9. Goos JDC, Teunissen CE, Veerhuis R, Verwey NA. Microbleeds relate to altered amyloid-beta metabolism in Alzheimer's disease. Neurobiol Aging. 2012; 33:1011.e1-1011.e9.

10. Noguchi-Shinohara M, Komatsu J, Samuraki M, Matsunari I, Ikeda T, Sakai K, et al. Cerebral Amyloid Angiopathy-Related Microbleeds and Cerebrospinal Fluid Biomarkers in Alzheimer's Disease. J Alzheimers Dis. 2017; 55:905-913. [PubMed: 27802236]

11. Rannikmäe K, Samarasekera N, Martînez-Gonzâlez NA, Al-Shahi Salman R, Sudlow CLM. Genetics of cerebral amyloid angiopathy: systematic review and meta-analysis. J Neurol Neurosurg Psychiatry. 2013; 84:901-908. [PubMed: 23457231]

12. Greenberg SM, Rebeck GW, Vonsattel JP, Gomez-Isla T, Hyman BT. Apolipoprotein E epsilon 4 and cerebral hemorrhage associated with amyloid angiopathy. Ann Neurol. 1995; 38:254-259. [PubMed: 7654074]

13. McCarron MO, Nicoll JA, Graham DI. A quartet of Down's syndrome, Alzheimer's disease, cerebral amyloid angiopathy, and cerebral haemorrhage: interacting genetic risk factors. J Neurol Neurosurg Psychiatry. 1998; 65:405-406. [PubMed: 9728967]

14. Alcolea D, Carmona-Iragui M, Suarez-Calvet M, Sánchez-Saudinos MB, Sala I, Antón-Aguirre S, et al. Relationship Between $\beta$-Secretase, Inflammation and Core Cerebrospinal Fluid Biomarkers for Alzheimer's Disease. J Alzheimers Dis. 2014; 42:157-167. [PubMed: 24820015]

15. Fortea J, Lladó A, Clarimón J, Lleó A, Oliva R, Peri J, et al. PICOGEN: five years experience with a genetic counselling program for dementia. Neurologia. 2011; 26:143-149. [PubMed: 21163230]

16. Carmona-Iragui M, Santos T, Videla S, Fernández S, Benejam B, Videla L, et al. Feasibility of lumbar puncture in the study of cerebrospinal fluid biomarkers for Alzheimer disease in subjects with Down syndrome. J Alzheimers Dis. 2017; 55:1489-1496. [PubMed: 27858714]

17. Rafii MS, Wishnek H, Brewer JB, Donohue MC, Ness S, Mobley WC, et al. The down syndrome biomarker initiative (DSBI) pilot: proof of concept for deep phenotyping of Alzheimer's disease biomarkers in down syndrome. Front Behav Neurosci. 2015; 9:239. [PubMed: 26441570]

18. Alcolea D, Martínez-Lage P, Sánchez-Juan P, Olazarán J, Antúnez C, Izagirre A, et al. Amyloid precursor protein metabolism and inflammation markers in preclinical Alzheimer disease. Neurology. 2015; 85:626-633. [PubMed: 26180139]

19. Antonell A, Fortea J, Rami L, Bosch B, Balasa M, Sánchez-Valle R, et al. Different profiles of Alzheimer's disease cerebrospinal fluid biomarkers in controls and subjects with subjective memory complaints. J Neural Transm. 2011; 118:259-262. [PubMed: 21161712]

20. Fazekas F, Chawluk JB, Alavi A, Hurtig HI, Zimmerman RA. MR signal abnormalities at $1.5 \mathrm{~T}$ in Alzheimer's dementia and normal aging. AJR Am J Roentgenol. 1987; 149:351-356. [PubMed: 3496763]

21. Scheltens P, Leys D, Barkhof F, Huglo D, Weinstein HC, Vermersch P, et al. Atrophy of medial temporal lobes on MRI in "probable" Alzheimer's disease and normal ageing: diagnostic value and neuropsychological correlates. J Neurol Neurosurg Psychiatry. 1992; 55:967-972. [PubMed: 1431963]

22. Yates PA, Villemagne VL, Ellis KA, Desmond PM, Masters CL, Rowe CC. Cerebral microbleeds: a review of clinical, genetic, and neuroimaging associations. Front Neurol. 2014; 4:205. [PubMed: 24432010] 
23. Ryan NS, Bastos-Leite AJ, Rohrer JD, Werring DJ, Fox NC, Rossor MN, et al. Cerebral microbleeds in familial Alzheimer's disease. Brain. 2012; 135:e201. author reply e202. [PubMed: 21685457]

24. Mok V, Wong KK, Xiong Y, Wong A, Schmidt R, Chu W, et al. Cortical and frontal atrophy are associated with cognitive impairment in age-related confluent white-matter lesion. J Neurol Neurosurg Psychiatry. 2011; 82:52-57. [PubMed: 20826875]

25. Gómez-Isla T, Growdon WB, McNamara MJ, Nochlin D, Bird TD, Arango JC, et al. The impact of different presenilin 1 andpresenilin 2 mutations on amyloid deposition, neurofibrillary changes and neuronal loss in the familial Alzheimer's disease brain: evidence for other phenotype-modifying factors. Brain. 1999:1709-1719. [PubMed: 10468510]

26. Yamada M. Predicting cerebral amyloid angiopathy-related intracerebral hemorrhages and other cerebrovascular disorders in Alzheimer's disease. Front Neurol. 2012; 3:64. [PubMed: 22539931]

27. Kirsch W, McAuley G, Holshouser B, Petersen F, Ayaz M, Vinters HV, et al. Serial susceptibility weighted MRI measures brain iron and microbleeds in dementia. J Alzheimers Dis. 2009; 17:599_ 609. [PubMed: 19433895]

28. Head E, Lott IT, Wilcock DM, Lemere CA. Aging in Down Syndrome and the Development of Alzheimer's Disease Neuropathology. Curr Alzheimer Res. 2016; 13:18-29. [PubMed: 26651341]

29. Buss L, Fisher E, Hardy J, Nizetic D, Groet J, Pulford L, et al. Intracerebral haemorrhage in Down syndrome: protected or predisposed? F1000Research. 2016; 5

30. Xiong L, Davidsdottir S, Reijmer YD, Shoamanesh A, Roongpiboonsopit D, Thanprasertsuk S, et al. Cognitive Profile and its Association with Neuroimaging Markers of Non-Demented Cerebral Amyloid Angiopathy Patients in a Stroke Unit. J Alzheimers Dis. 2016; 52:171-178. [PubMed: 27060947]

31. Kim M, Bae HJ, Lee J, Kang L, Lee S, Kim S, et al. APOE epsilon2/epsilon4 polymorphism and cerebral microbleeds on gradient-echo MRI. Neurology. 2005; 65:1474-1475. [PubMed: 16275840]

32. Lemere CA, Blusztajn JK, Yamaguchi H, Wisniewski TM, Saido TC, Selkoe DJ. Sequence of deposition of heterogeneous amyloid beta-peptides and APO E in Down syndrome: implications for initial events in amyloid plaque formation. Neurobiol Dis. 1996; 3:16-32. [PubMed: 9173910]

33. van Etten ES, Verbeek MM, van der Grond J, Zielman R, van Rooden S, van Zwet EW, et al. $\beta$ Amyloid in CSF: Biomarker for preclinical cerebral amyloid angiopathy. Neurology. 2017; 88:169-176. [PubMed: 27903811]

34. Sperling, Ra, Aisen, PS., Beckett, La, Bennett, Da, Craft, S., Fagan, AM., et al. Toward defining the preclinical stages of Alzheimer's disease: recommendations from the National Institute on Aging-Alzheimer's Association workgroups on diagnostic guidelines for Alzheimer's disease. Alzheimers Dement. 2011; 7:280-292. [PubMed: 21514248]

35. Sperling R, Salloway S, Brooks DJ, Tampieri D, Barakos J, Fox NC, et al. Amyloid-related imaging abnormalities in patients with Alzheimer's disease treated with bapineuzumab: a retrospective analysis. Lancet Neurol. 2012; 11:241-249. [PubMed: 22305802]

36. Boche D, Zotova E, Weller RO, Love S, Neal JW, Pickering RM, et al. Consequence of A $\beta$ immunization on the vasculature of human Alzheimer's disease brain. Brain. 2008; 131:32993310. [PubMed: 18953056]

37. Sevigny J, Chiao P, Bussière T, Weinreb PH, Williams L, Maier M, et al. The antibody aducanumab reduces A $\beta$ plaques in Alzheimer's disease. Nature. 2016; 537:50-56. [PubMed: 27582220]

38. Pfeifer L, White L, Ross G, Petrovitch H, Launer L. Cerebral amyloid angiopathy and cognitive function: the HAAS autopsy study. Neurology. 2002; 58:1629-1634. [PubMed: 12058090]

39. Goos JDC, van der Flier WM, Knol DL, Pouwels PJW, Scheltens P, Barkhof F, et al. Clinical relevance of improved microbleed detection by susceptibility-weighted magnetic resonance imaging. Stroke. 2011; 42:1894-1900. [PubMed: 21566235]

\section{Abbreviations}

ADAD Autosomal dominant Alzheimer's disease 

APOE Apolipoprotein-E
APP Amyloid precursor protein
ARIA Amyloid-related imaging abnormalities
CAA Cerebral amyloid angiopathy

CAMCOG-DSambridge Examination for mental Disorders of Older People with DS and Others with Intellectual Disabilities-Cognitive Scale

cSS Cortical superficial siderosis

d-EOAD Dementia in early onset Alzheimer's disease

DABNI Down Alzheimer Barcelona Neuroimaging Initiative

dAD-DS Alzheimer's disease dementia in Down syndrome

dADAD Dementia in autosomal dominant Alzheimer's disease

DS Down syndrome

DSBI Down syndrome biomarker initiative

EOAD Early onset Alzheimer's disease

FLAIR Fluid attenuation inversion recovery

GRE Gradient echo

ICH Intracerebral hemorrhage

mBCAA Modified Boston criteria for cerebral amyloid angiopathy

MMSE Mini-mental state examination

MTA Medial temporal atrophy

p-EOAD Prodromal Alzheimer's disease in early onset Alzheimer's disease

pAD-ADAD Prodromal Alzheimer's disease in autosomal dominant Alzheimer's disease

pAD-DS Prodromal Alzheimer's disease in Down syndrome

PSEN1 Presenilin 1

PSEN2 Presenilin 1

SWI Susceptibility weighted imaging

WMH White matter hyperintensities 

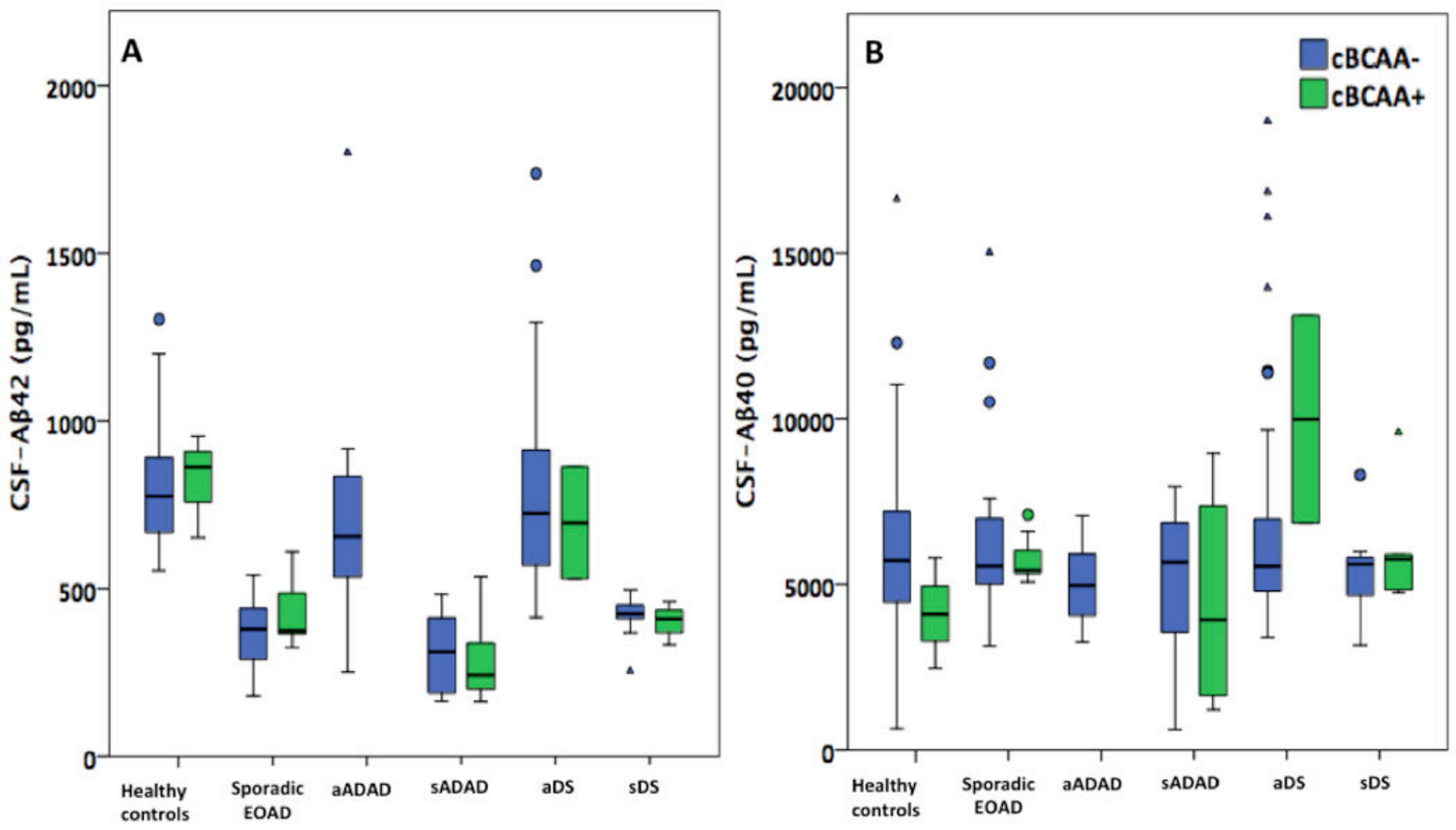

Figure 1. CSF biomarker levels in the clinical groups, according to modified Boston criteria for CAA (cBCAA)

Box plot displaying the distribution of CSF-A $\beta 42$ (A) and CSF-A $\beta 40$ (B) from healthy controls, sporadic EOAD, asymptomatic ADAD (aADAD), symptomatic ADAD (sADAD), asymptomatic DS (aDS), and symptomatic DS (sDS). Subgroups in blue represent those subjects who do not fulfill cBCAA, subgroups coloured in green do fulfill cBCAA for possible or probable CAA.

No differences in levels of CSF-A $\beta 42$ or in CSF- A $\beta 40$ were detected between subjects that fulfilled cBCAA and those who did not within each clinical group. 
A) Modified Boston criteria

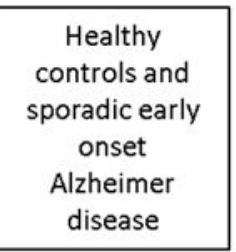

Autosomal dominant Alzheimer disease

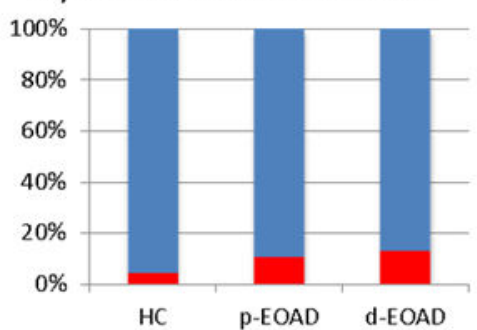
and medial temporal lobe atrophy
B) Fazekas score
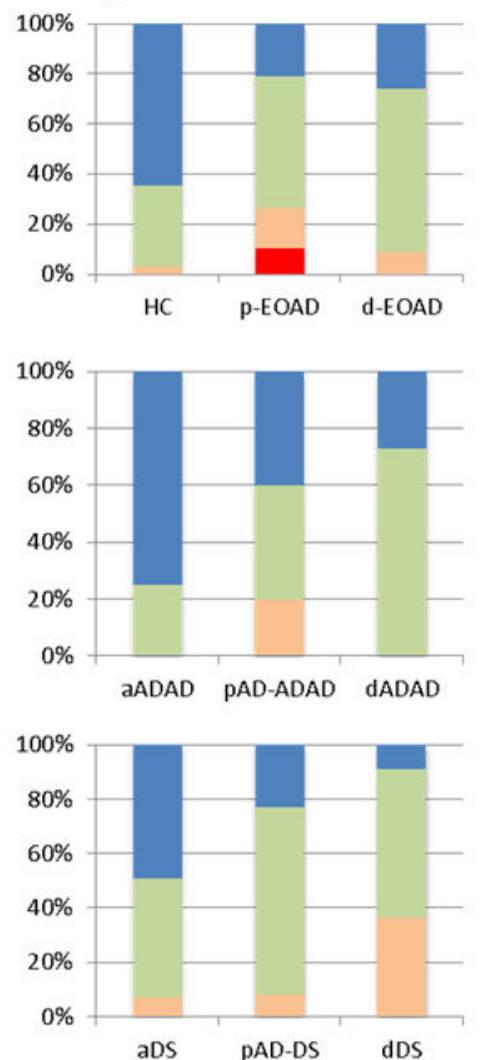

mscore 0 in score 1 score 2 score 3
C) Medial temporal atrophy
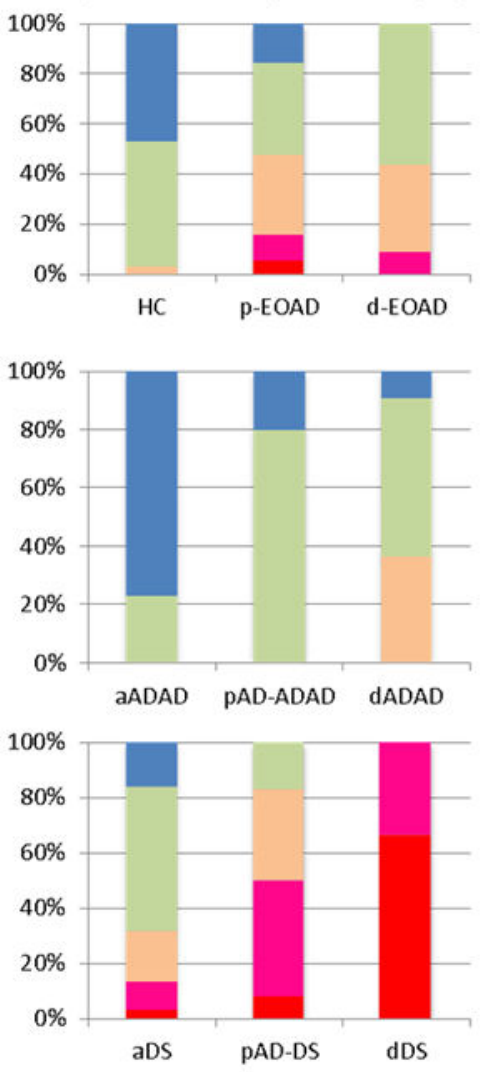

"MTA $0=$ MTA $1=$ MTA2

- MTA $3=$ MTA 4

Figure 2. Proportion of subjects in each group according modified Boston criteria, Fazekas score,

Frequency bar graph showing: A) modified Boston criteria fulfillment, B) Fazekas score and, C) MTA score of each clinical group. Healthy controls and sporadic AD in the first row, ADAD in the second row, DS in the third row. 

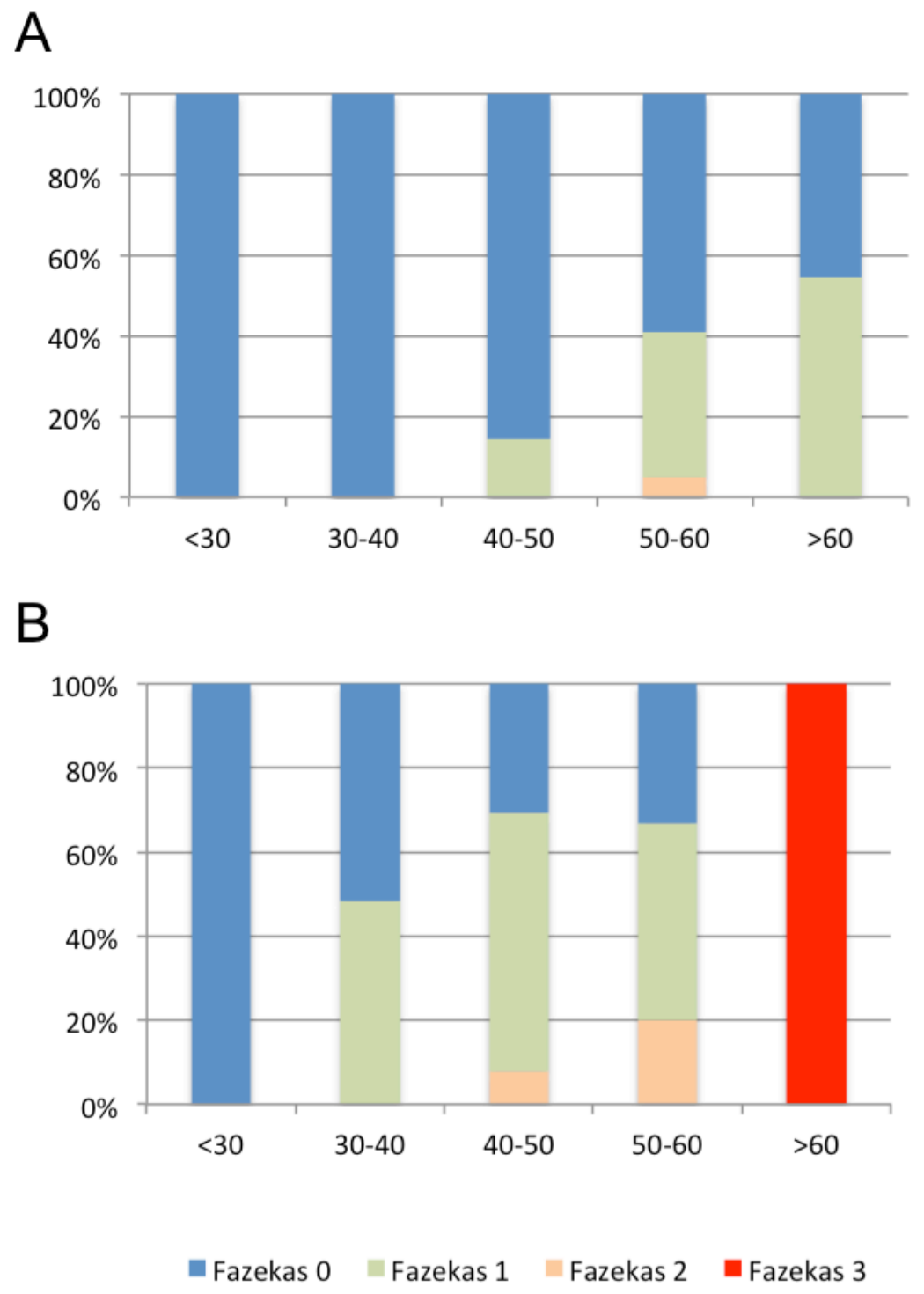

Figure 3. Proportion of subjects according to Fazekas score by range of age

The frequency bar graphs showing the percentage of subjects with each Fazekas categories by age in: A) Healthy controls and, B) asymptomatic Down syndrome subjects. 







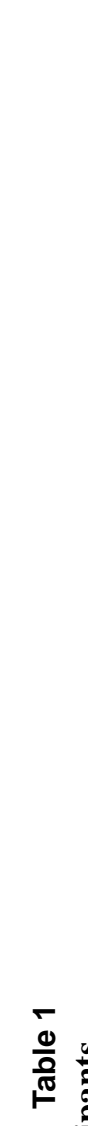

롬

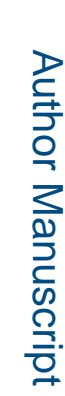


